\title{
Multidimensional Sample Surface Analysis by AFM-in-SEM
}

Veronika Novotna, Veronika Hegrova, Josef Horak, Zdenek Novacek, Michal Pavera and Jan Neuman

NenoVision, Brno, Jihomoravsky kraj, Czech Republic

Correlative imaging was pioneered by combining light and electron microscopy, herein we present an approach for coupling scanning electron microscope (SEM) with an atomic force microscope (AFM) into one tool for advanced surface analysis in the (sub)nanometer range. Resulting correlative workflow benefits from the complementarity of both techniques alongside significant savings both in time and resources: the AFM can provide information about the topography, roughness, mechanical, magnetic and electric properties of the sample surface while scanning electron microscopy (SEM) possesses a great material contrast, large depth of focus, fast imaging and further possibilities depending on its accessories.

The NenoVision company achieved that by developing a compact AFM, LiteScope ${ }^{\mathrm{TM}}$, designed for fast and easy integration into most of the SEM systems. LiteScope's unique design allows for simultaneous acquisition and correlation of SEM and AFM data through patented technology called Correlative Probe and Electron Microscopy $\left(\mathrm{CPEM}^{\mathrm{TM}}\right)$ [1]. Currently, CPEM can accommodate information from the above-mentioned AFM techniques together with secondary electrons (SE), backscattered electrons (BSE), electron beam induced current (EBIC) and cathodoluminescence (CL). Using CPEM $^{\mathrm{TM}}$, the electron beam is kept still (i.e. point mode, spectroscopy mode) and focused close to the AFM tip, while scanning is done just by a piezo scanner with the sample. Distance (offset) between the AFM tip and electron beam is constant during the whole measurement, which enables precise data correlation into one 3D image (Figure 1).

Another key advantage of the AFM-in-SEM approach is the in-situ measurement, meaning minimal sample handling and contamination due to vacuum preservation inside the SEM, which is crucial for sensitive samples. LiteScope can be tilted and is thus compatible with a focused ion beam (FIB) and gas injection system (GIS); this opens possibilities for immediate topographical analysis of the modified surface and also for advanced FIB/GIS process optimization.

AFM-in-SEM technology finds its place in many applications in the field of material science, nanotechnology, semiconductors, and life sciences. Two applications were chosen to show the strengths of the CPEM correlative technique, but also the necessity of the AFM-in-SEM concept. 2D materials are represented by study exfoliated WSe2 flakes on Si nanopillars to create a single photon emitter (Figure 1). The SEM provided fast microstructure localization and great material contrast, while the AFM enabled topographical monolayer resolution. The correlated 3D CPEM view provides a better understanding of the obtained data since a single-photon emitter is created only by a certain shape of the WSe2 monolayer over the pillars.

Figure 2 depicts the investigation of local electrical properties of conductive particles in a nonconductive matrix, an application that would be unfeasible by separate SEM and AFM techniques. The presented conductive CPEM (C-CPEM) technique is an extension of the common conductive AFM (C-AFM) since the electron beam at the constant distance from the tip replaces the need for an applied bias in the insulated areas, and thus, makes those visible. 
Taken together, the AFM-in-SEM approach opens completely new possibilities for advanced data correlation that enables to extract more scientific insight and to perform measurements that would have not been possible by the separate imaging techniques.
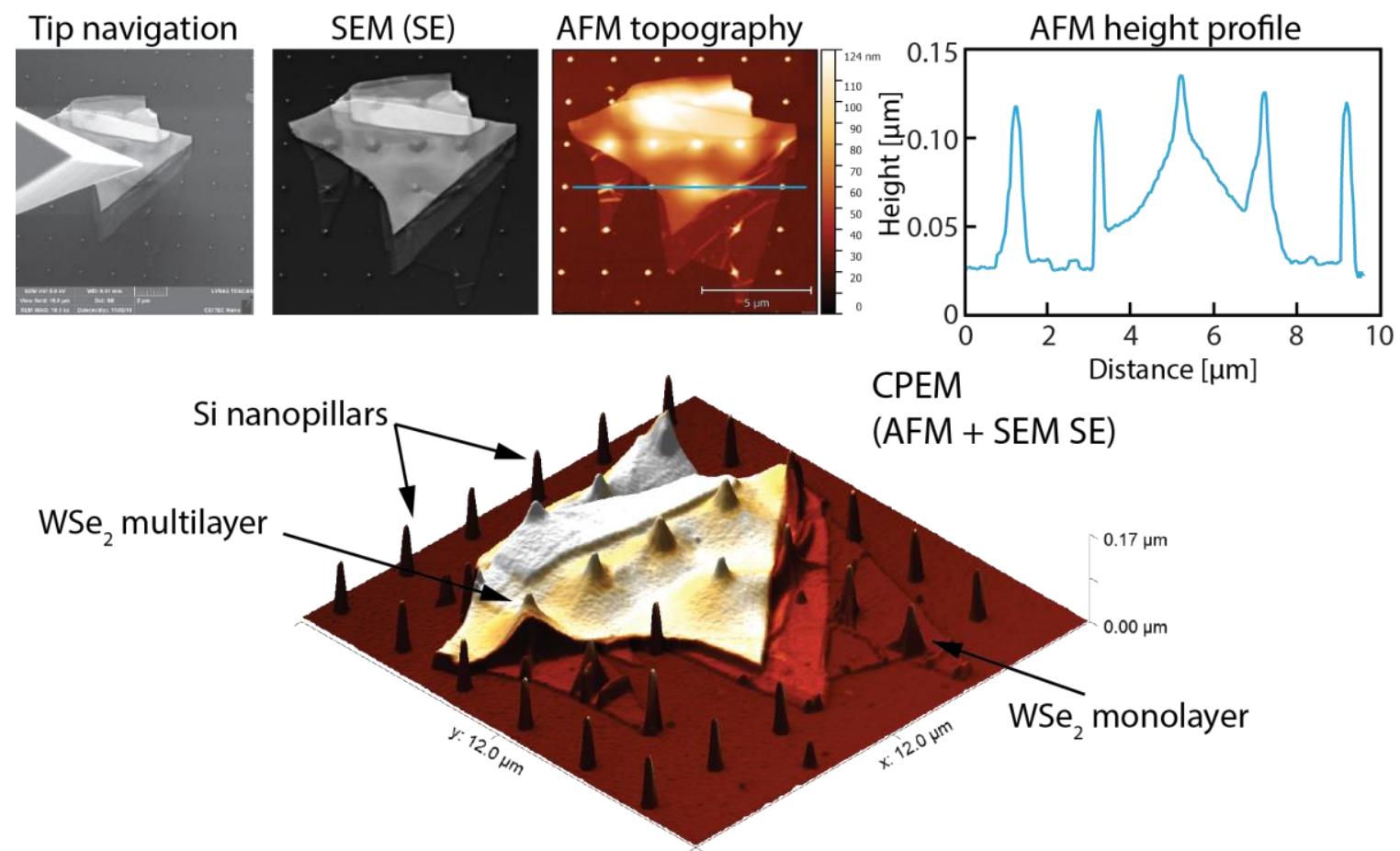

Figure 1. Exfoliated WSe2 flakes on Si nanopillars: Overview of the sample in contact with the AFM tip. The signal from secondary electrons detector (SE), AFM topography with a height profile, and 3D CPEM view of correlated AFM topography and SEM signal.
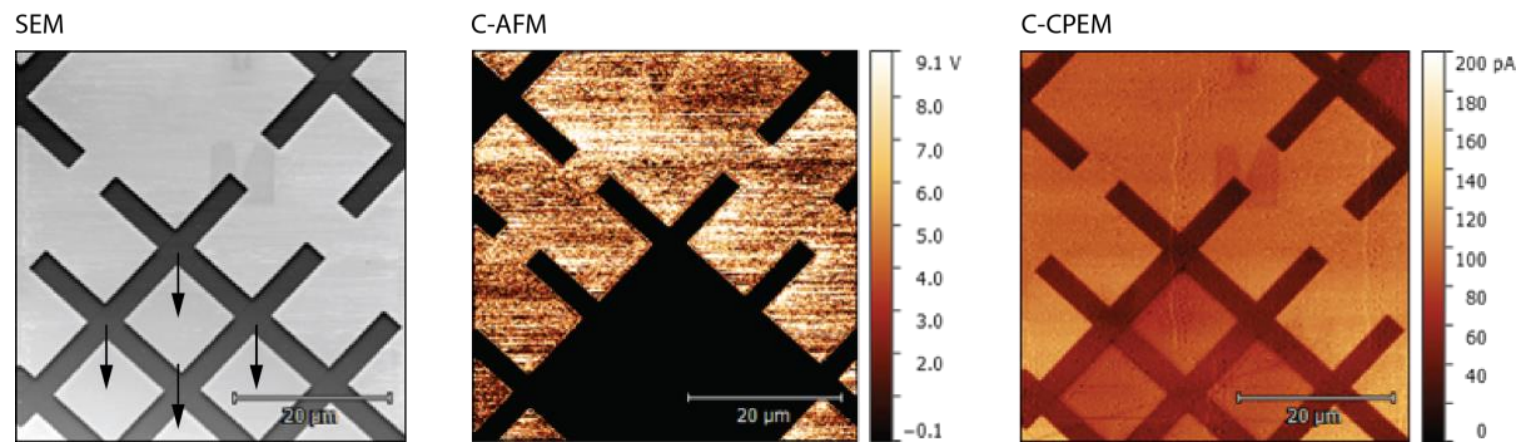

Figure 2. The same sample area imaged by SEM showing the insulated areas (highlighted by arrows) that failed to be visualized in the C-AFM conductivity map (dark area), but are clearly visible by the C-CPEM technique using the SEM electron beam as a bias source.

References

[1] J Neuman, Z Novacek, M Pavera and V Novotna, Microscopy and Microanalysis, 25(S2) (2019), p. 430-431. 